\title{
EL CONSUMIDOR COMO ACTOR PRINCIPAL EN LA CULTURA DEL RECICLAJE
}

\author{
Lic. Adriana Gabriela Cahue Olvera ${ }^{1}$
}

Dra. María Angélica Cruz Reyes ${ }^{2}$

\section{Resumen}

La generación de residuos sólidos (RS) es inherente al consumo de productos protegidos con diversos tipos de empaque, entre éstos se encuentran los alimentos procesados. El consumo de alimentos es una actividad básica de los seres humanos, quienes buscan satisfacer sus necesidades a través de productos y/o servicios. Sin embargo, conforme las personas consumen y se ven influenciadas por otros factores, se genera una reconversión a los deseos y se da pauta a lo que se conoce como consumismo. El consumismo ha generado grandes flujos de materiales de desecho, de tal manera que es visible la problemática social derivada, ya que ocasiona la explotación de materias primas, agotamiento de los recursos, la pérdida de calidad del ambiente y calidad de vida.

El presente trabajo, tiene como objetivo analizar cuáles son los factores que propician la cultura del reciclaje en los consumidores de alimentos procesados, mediante el proceso de observación y análisis de información estadística sobre los programas de reciclaje que ha diseñado el gobierno de la Ciudad de México, y si es que éstos generan una concientización en la administración de los RS entre los consumidores de productos procesados, así como la perspectiva del consumidor respecto al cambio de hábitos y beneficios sociales obtenidos.

Palabras clave: Consumidor, residuos sólidos, cultura de reciclaje, sustentabilidad.

\footnotetext{
1 Licenciada en Relaciones Comerciales y alumna de maestría Instituto Politécnico Nacional, ESCA Tepepan.

2 Profesora - Investigadora Instituto Politécnico Nacional, ESCA Tepepan.
} 


\section{Abstract}

The generation of solid waste (RS) is inherent to the consumption of products protected with various types of packaging, generally the products that are most protected are processed foods. The consumption of food is a basic base of human beings, who seek to satisfy their needs through products and services; However, with these human beings they are consumed and influenced by other factors, a reconversion to the desires is generated and the pattern is given to what is known as consumerism. Consumption has generated large flows of waste materials, in such a way that the social problems arising are visible since they cause the exploitation of raw materials, the depletion of resources, the loss of quality of the environment and the quality of life.

The objective of this paper is to analyze the factors that favor the culture of recycling in consumers of processed foods, through the process of observation and analysis of statistical information on recycling programs designed by the government of Mexico City. and if so, there is an awareness in the management of the RS among the consumers of processed products, as well as the perspective of the consumer regarding the change of habits and social benefits obtained.

Keywords: Consumer, solid waste, recycling culture, sustainability.

\section{El consumidor y la generación de residuos sólidos}

La actividad humana constreñida por los actos económicos, genera diariamente una gran cantidad de residuos, los cuales crean un problema complejo de contaminación, además de involucrar aspectos como la tecnología, la innovación y la utilidad, ambiente, y actores como la empresa, la sociedad y los distintos niveles de gobierno. Al hacer frente a la problemática de la contaminación desde una postura de normativa en la Ciudad de México, el manejo de los residuos sólidos está sujeto a la Ley General para la prevención y gestión integral de los residuos.

Los residuos sólidos urbanos (RSU) se definen como "aquellos generados directamente por el consumo en casas habitación, resultantes 
del desecho de materiales utilizados en sus actividades domésticas, de los productos que consumen, sus envases, embalajes y empaques" (Ley General para la prevención y gestión integral de los residuos, 2003). Y se clasifican en tres categorías: 1. Residuos peligrosos; 2 . Residuos de manejo especial y 3 . Residuos sólidos urbanos.

Los RSU tienen relación directa con el consumidor y sus actividades cotidianas, los consumidores son aquellas personas que utilizan las mercancías o artículos que compran, ya sean bienes o servicios produ$\operatorname{cidos}^{3}$ que una vez satisfecha su necesidad, y utilizados los productos, sus envases o contenedores son desechados, comenzando así la acumulación de RSU.

Desde esta perspectiva, la generación de RSU depende de los hábitos de consumo y de diversos factores que promueven la generación de residuos sólidos urbanos, tales como la corta duración de los bienes, la ineficiencia en procesos productivos, estrategias de mercado, el ciclo del producto, entre otros más que en conjunto influyen en los patrones de compra de los consumidores y conducen a un estilo de vida consumista.

Con la finalidad de enmarcar la diferencia entre consumo y consumismo, la PROFECO puntualiza que

el consumismo, es el resultado de la reconversión de los deseos, ganas o anhelos humanos, en el que la sociedad influye como parte de la integración social, las capas sociales y la formación del individuo. A diferencia del consumo, que es un rasgo y una ocupación del individuo humano, el consumismo es un atributo de la sociedad.

Derivado del consumismo, los RSU han colocado a México dentro de los primeros lugares de generación de residuos en América Latina (SEMARNAT. Fundación Flor y Canto, 2012). Particularmente en la Ciudad de México la generación de RSU diaria asciende a 12843 toneladas de

3 Definición de consumidor propuesta por Mercado, Salvador (2011): Consumidor es aquel que utiliza las mercancías o artículos que compra, ya sean bienes o servicios producidos. 
basura, de las cuales el 48\% proviene de los hogares. Es importante resaltar que los RSU provenientes de hogares no son recolectados al 100\% por los servicios de limpia y ,sin embargo, a través de éstos se procesan 297.04 toneladas diarias, integradas en un $53 \%$ por plástico, papel y sus derivados, $0.14 \%$ envases de vidrio y 0.001 por latas (Inventario de Residuos Sólidos de la Ciudad de México, 2015).

Los datos anteriores permiten deliberar que los empaques de los alimentos procesados generan un gran volumen de residuos y no son correctamente tratados, quizá, y entre otros factores, por la falta de cultura de reciclaje de los consumidores.

En tal sentido, puede resultar fácil considerar que los consumidores pueden ser pieza clave para la reducción de residuos a través de cambios en sus preferencias de consumo y hábitos sobre del manejo de los materiales que se emplean en los diversos actos de producción y comercio; aunque, el manejo correcto de los RSU también involucra a los distintos órganos de gobierno y empresas productoras de alimentos procesados.

\section{Cultura del reciclaje}

Para profundizar en el tema de la cultura del reciclaje, es necesario hacer una separación de conceptos; por un lado, de manera general, la UNESCO concibe a la cultura como el conjunto de "rasgos distintivos, espirituales y materiales, intelectuales y afectivos que caracterizan una sociedad o un grupo social"4, estos rasgos a su vez pueden ser utilizados para promover el desarrollo y alcanzar el bienestar.

4 Dentro de la Conferencia sobre las políticas culturales de 1982 la UNESCO declara: la cultura [...] engloba, además de las artes y las letras, los modos de vida, los derechos fundamentales al ser humano, los sistemas de valores, las tradiciones y las creencias y que la cultura da al hombre la capacidad de reflexionar sobre sí mismo. Es ella la que hace de nosotros seres específicamente humanos, racionales, críticos y éticamente comprometidos. A través de ella discernimos los valores y efectuamos opciones. A través de ella el hombre se expresa, toma conciencia de sí mismo, se reconoce como un proyecto inacabado, pone en cuestión sus propias realizaciones, busca incansablemente nuevas significaciones, y crea obras que lo trascienden. 
Por otra parte, Boas, citado por Martínez-Hernáez (El dibujante de límites: Franz Boas y la (im)posibilidad del concepto de cultura antropológica, 2010), menciona que la cultura es

todo lo que incluye las manifestaciones de los hábitos sociales de una comunidad, las reacciones del individuo en la medida en que se ven afectadas por las costumbres del grupo en que vive, y los productos de las actividades humanas en la medida en que se ven determinadas por dichas costumbres 5 .

Ahora bien, desde el punto de vista organizacional, Hofstede define la cultura como "la programación mental colectiva que distingue a los miembros de una organización de otra" $"$ en donde, al ser un elemento intangible, se presentan diversos indicadores y manifestaciones culturales (Franklin, 2011), tales como la moral y la ética, los perfiles socioculturales y la ideología de la organización.

Por lo descrito anteriormente, la cultura de una organización, puede estimular la conducta de los miembros que la integran en el momento en que las partes interesadas internas aceptan y se comprometen a dicha cultura (valores e ideologías). Una manera de externalizar esta cultura es mediante la visión de la organización, que se comunique mediante el producto y sus estrategias de comercialización. Una empresa comprometida con el cuidado del medio ambiente generalmente traerá consigo personal involucrado en estos ámbitos, para generar acciones dirigidas que promuevan tanto interna como externamente el cuidado del ambiente y de lo social.

Adicionalmente, el reciclaje puede ser concebido como el "procedimiento mediante el cual se somete una materia o un producto ya utili-

5 Martínez-Hernáez hace un análisis de la obra de Franz Boas y una recopilación histórica del concepto de cultura, destacando el definido por Boas.

6 A través del análisis de algunas definiciones occidentales y antropológicas Hofstede define cultura como "la programación mental colectiva que distingue a los miembros de un grupo o categoría", la cual es colectiva por ser compartida con personas que han vivido en el mismo entorno social. 
zado a un tratamiento total o parcial que origina un producto totalmente nuevo que puede ser reintegrado al mercado en términos económicos"7 (Cultura de reutilización y reciclaje en estudiantes de humanidades de primer y tercer grados, 2010).

A partir de estas definiciones, es posible vislumbrar la cultura del reciclaje como una alternativa para generar un bienestar social a través de la concientización y adopción de una correcta postura por parte de los consumidores, para aprovechar al máximo los beneficios de los materiales utilizados. Y de esta forma disminuir el impacto ecológico que éstos puedan generar, de tal manera que, a través de los rasgos distintivos de una comunidad, marcados por sus hábitos, costumbres, actividades y la influencia ejercida por las organizaciones hacia su personal y consumidores, propicien elementos para fomentar la cultura del reciclaje.

Sin embargo, esta cultura debe ser reforzada mediante la intervención de organismos, tanto públicos como privados, incluyendo a quienes generan las políticas públicas que a través de estudios, de la difusión de información, de la promulgación de leyes y políticas, propaguen en los consumidores un mayor grado de concientización y educación en los temas concernientes al reciclaje y generación de residuos.

\section{El reciclaje en la Ciudad de México y su impacto}

Con la finalidad de hacer frente al incremento de RSU el gobiero de la Ciudad de México crea un conjunto de políticas públicas, las cuales, de acuerdo con Roth (2002), son

un conjunto de varios objetivos colectivos considerados necesarios o deseables y por medios y acciones que son tratados por lo menos parcialmente por una institución u organización gubernamental con la finalidad de orientar el comportamiento de actores

7 Rodríguez, Adrián, Antonio, Jorge y Ávalos, Karla, proponen esta definición de reciclaje dentro de su trabajo "Cultura de reutilización y reciclaje en estudiantes de humanidades de primer y tercer grados". 
individuales o colectivos para modificar una situación percibida como insatisfactoria o problemática ${ }^{8}$.

Como parte de una política regulatoria, la Ley General para la Prevención y Gestión Integral de los Residuos (LGPGIR), establece que tanto los productores, comercializadores, consumidores y autoridades de gobierno son fundamentales para que el manejo de los residuos sea eficiente, viable y factible (Gaceta Oficial de la Ciudad de México, 2016).

En la Ciudad de México, el 1 de octubre de 2004, se creó el primer programa de Gestión de los Residuos Sólidos al cobijo de la LGPGIR con la finalidad de establecer la separación de los residuos en orgánicos e inorgánicos y un programa de limpia y manejo de residuos. Posteriormente, el 13 de septiembre de 2010 se identificaron las áreas de oportunidad y se estableció el Programa de Gestión Integral de los Residuos Sólidos (PGIRS).

El PGIRS integra las estrategias, metas y acciones necesarias para llevar a cabo el manejo adecuado de los residuos sólidos de esta Ciudad de México, bajo los siguientes criterios:

- Reducción de la generación de los residuos sólidos.

- $\quad$ Separación en la fuente.

- Reincorporación al ciclo productivo de materiales reutilizables o reciclables.

- Empleo de infraestructura para su adecuado manejo.

- Promoción de la cultura, educación y capacitación ambiental.

8 En 2002 RothDeubel, A.N. a partir de diversos conceptos define las políticas públicas como un conjunto conformado por uno o varios objetivos colectivos considerados necesarios o deseables y por medios y acciones que son tratados por lo menos parcialmente por una institución u organización gubernamental con la finalidad de orientar el comportamiento de actores individuales o colectivos para modificar una situación percibida como insatisfactoria o problemática, teniendo como objeto de estudio los objetivos que el estado considera deseables, los medios y acciones procesados y los resultados de dichas acciones. 
Adicionalmente, el PGIRS, a partir del diagnóstico y evaluación, propone estrategias para incidir en la modificación de los hábitos de la población sobre la generación y manejo de sus residuos a través del fomento del reciclaje de los residuos sólidos generados, por medio de de la creación o fortalecimiento de mercados de materiales potencialmente valorizables.

Anualmente las actividades del PGIRS son evaluadas de manera integral mediante el Inventario de Residuos Sólidos de la Ciudad de México, con la finalidad de concentrar información, identificar las principales fuentes de generación del comportamiento en la prestación del servicio de recolección de residuos de manera separada, la infraestructura con que se cuenta para el manejo integral de los residuos y las cantidades que se manejan en ella, así como la información que se obtiene a través de los planes de manejo de residuos sólidos (Programa para la Gestión Integral de Residuos Sólidos, 2016).

El PGIRS cuenta con diversos planes para el manejo de RSU con el fin de alcanzar la meta de "basura cero" a través del tratamiento y aprovechamiento de los RSU, en donde se busca realizar un cambio en la perspectiva del manejo de residuos sólidos y así priorizar el fortalecimiento de la prevención y minimización en la generación de Residuos.

\section{Figura 1. Estrategia de "Basura Cero"}

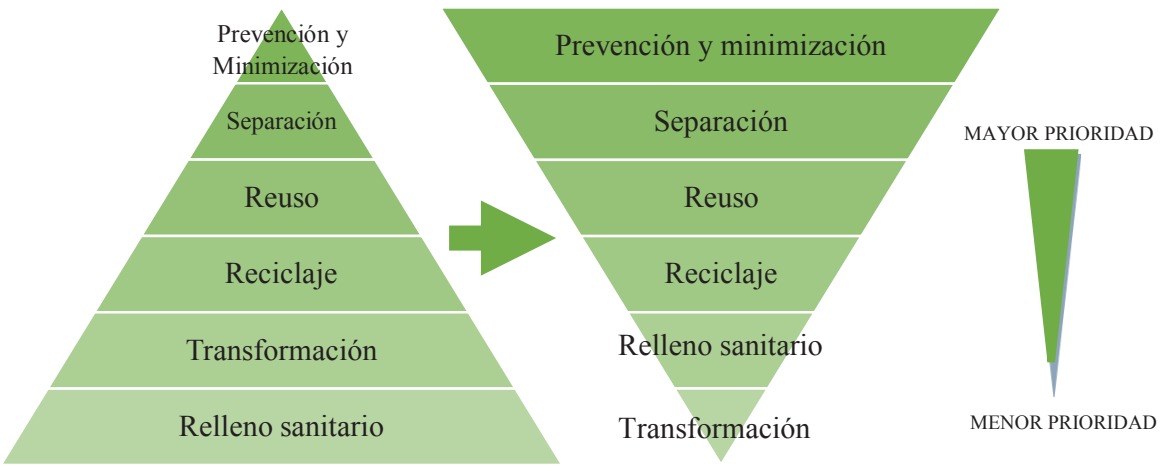

Fuente: Programa de Gestión Integral de Residuos Sólidos, 2016

De esta manera, a través de las políticas públicas, aplicadas a programas de reciclaje, el gobierno de la Ciudad de México busca crear conciencia 
respecto a la relevancia del consumo y separación de residuos valorizables o reciclables, dando énfasis en fomentar la prevención y minimización de la generación de RSU (consumo responsable) hasta lograr su transformación.

\section{Gestión integral de los programas de reciclaje}

Los programas de gestión integral de los residuos sólidos evalúan el manejo de los residuos sólidos, identificando las áreas de oportunidad que deberían bordarse de forma contundente y proporcionando una visión estratégica para la planeación de la gestión integral de los residuos sólidos en la Ciudad de México.

Tal y como ha sido mencionado con anterioridad, el 48\% de los RSU proviene de los hogares de la Ciudad de México, es decir, son el resultado del desecho de los empaques de productos procesados que en algún momento fueron utilizados para satisfacer sus necesidades.

Los envases de los alimentos procesados, con la finalidad de conservar los alimentos, se encuentran elaborados principalmente de derivados del papel y el cartón, como es el caso de los empaques tetrapack, plástico, vidrio y enlatados. Todos estos materiales, al ser desechados, pueden ser valorizados como reciclables y tener un reuso o tratamiento especial que ayude significativamente a la disminución de contaminación ambiental.

El gobierno de la Ciudad de México, basado en el estilo consumista de las personas, cuenta particularmente con tres programas de recolección de RSU, de los cuales, dos de ellos se enfocan en el tratamiento de RSU especiales como las pilas y aparatos eléctricos, mientras que el tercer programa, se encuentra enfocado en el aprovechamiento de los materiales que son principalmente producto del consumo de alimentos procesados.

\section{Ponte las Pilas}

Este programa ofrece a los consumidores una alternativa para la correcta disposición de las pilas mediante el depósito de éstos en columnas 
recolectoras ubicadas en más de 400 puntos de la Ciudad de México. Considerando que el $30 \%$ de las pilas están constituidas por materiales tóxicos como mercurio, plomo, litio, cadmio y níquel, "Ponte las pilas" pretende educar y generar una concientización para el desecho de las pilas y dar una alternativa correcta al reciclaje de este tipo de materiales.

De acuerdo con Greenpeace (Información Básica sobre Pilas y Baterías, 2005), en México se desechan anualmente 35,500,000 kilos de pilas y baterías, que de acuerdo a su composición, puede tener diversos efectos contaminantes, por ejemplo: una pila de mercurio puede contaminar 600 mil litros de agua; mientras que una de zinc-aire, 12 mil litros; una de óxido de plata, 14 mil litros; una alcalina, 167 mil litros de agua y una pila de carbón-zinc 3 mil litros. Asimismo, los materiales con los que son fabricadas las pilas pueden tener efectos nocivos sobre la salud:

- Mercurio: Es un posible cancerígeno y es bioacumulable ya que contamina el agua y la tierra, con lo que puede llegar a la comida e incluso se acumula en los tejidos de peces.

- Plomo: Puede dañar el sistema nervioso, los riñones y el sistema reproductivo y no se degrada, lo cual provoca que se adhieran partículas en el suelo y puede pasar a aguas subterráneas.

- Litio: Daña al sistema nervioso y fácilmente se filtra a los mantos acuíferos.

- Cadmio: El cadmio entra al aire y al agua de fuentes como vertederos y derrames de desechos domésticos, puede viajar largas distancias y una vez que éste llega a los alimentos, puede provocar efectos como irritación en el estómago, vómitos y diarrea.

- Níquel: Se libera a la atmósfera principalmente por los incineradores de basura, una vez en el aire, se adhiere a partículas de polvo que se depositan en el suelo, un contacto constante con este tipo de sustancias, puede producir enfermedades respiratorias.

Por lo nocivo de las pilas y el impacto a la salud, el programa dispone de 400 columnas de acopio en las vialidades de la Ciudad. Quincenalmente son recolectadas, almacenadas y enviadas a un centro de recicla- 
je que recupera la carcasa de las pilas para la fabricación de alambre, reutilizar metales (litio, cadmio, níquel y zinc) y aprovechar los materiales restantes como estabilizadores de residuos peligrosos.

\section{Reciclatrón}

De acuerdo con la Ley de Residuos Sólidos del Distrito Federal, los residuos electrónicos y eléctricos también requieren un plan de manejo especial para aprovechar su valor y gestionar su disposición final de manera ambientalmente adecuada y controlada. De acuerdo con la Secretaría del Medio Ambiente del Distrito Federal, en 2015 (Inventario de Residuos Sólidos de la Ciudad de México) se generaron en nuestro país alrededor de 358 mil toneladas de este tipo de residuos electrónicos, lo que nos proporciona un indicador de $3.2 \mathrm{~kg}$ per cápita, de los cuales únicamente se recicla el 10\%, mientras que un $40 \%$ permanece almacenado en casas y bodegas, el 50\% restante, llega a estaciones de transferencia o a manos de recicladores informales (chatarreros), rellenos sanitarios o tiraderos no controlados.

En la Ciudad de México, la disposición de residuos electrónicos se encuentra distribuida de la siguiente manera: $42 \%$ de los residuos son entregados al camión de la basura, 30\% los regala, 11\% los almacena, y el 17\% los vende (Inventario de Residuos Sólidos de la Ciudad de México, 2015). Con la finalidad de atender esta problemática, el Reciclatrón pretende que los consumidores realicen la correcta disposición de residuos electrónicos al propiciar el conocimiento e impacto de clasificar los residuos electrónicos mediante el acopio en espacios públicos, escuelas y/o explanadas. En cada una de las jornadas mensuales se instalan centros de recepción de residuos que se organizan en 4 categorías (A, B, C, y D) para gestionar el tratamiento y disposición adecuados. 
Tabla 1. Clasificación de residuos electrónicos.

\begin{tabular}{|l|l|}
\hline Categoría A & $\begin{array}{l}\text { Teclados, impresoras, faxes, MP3, cámaras, escáner, radiograba- } \\
\text { doras, teléfonos, electrodomésticos }\end{array}$ \\
\hline Categoría B & $\begin{array}{l}\text { CPUs, monitores, laptops, mini laptops, discos duros, tarjetas y } \\
\text { televisiones. }\end{array}$ \\
\hline Categoría C & Celulares y pilas \\
\hline Categoría D & Cargadores, cables, discos y películas \\
\hline
\end{tabular}

Fuente: Programa para la Gestión Integral de Residuos Sólidos, 2016

\section{Mercado de trueque}

Partiendo del hecho de que diariamente se generan más de 12 mil toneladas de desechos y que dentro de éstos hay una gran cantidad de materiales reciclables, surge el mercado del trueque como un programa de intercambio que busca concientizar a los consumidores respecto a la separación y aprovechamiento de RSU, mientras impulsa el desarrollo y consumo de productos locales.

Los consumidores participantes en el programa asisten con los residuos recolectados, separados (papel, botellas de vidrio, cartón, latas de aluminio, PET, tetrapack y electrónicos) y limpios, los cuales son valorados y canjeados por "puntos verdes", con los que los participantes se hacen acreedores de productos agrícolas como verduras, hortalizas, moles, quesos, plantas de ornato, semillas, hierbas aromáticas y dulces típicos.

Los productos agrícolas provienen de Xochimilco, Tláhuac, Milpa Alta y otras zonas agrícolas de la Ciudad de México. El propósito es fomentar y apoyar a los productores del suelo de conservación con el objetivo de concientizar a la población sobre la oferta local que existe y resaltar que consumir de manera local tiene un impacto directo en la disminución de la huella de carbono, además de propiciar el comercio justo y el uso de métodos tradicionales en la Ciudad. 


\section{Análisis de la información}

El presente trabajo tiene un alcance descriptivo con información a priori, se observaron los datos de los años 2013 a 2016, para analizar cuáles son los factores que propician la cultura del reciclaje en los consumidores de alimentos procesados y alcanzar el objetivo trabajando con las estadísticas anuales de los tres programas citados.

Así el programa "ponte las pilas", desde su implementación, ha tenido una respuesta constante ya que es permanente y de fácil acceso a la población de la Ciudad de México, de acuerdo con los inventarios de RSU de la Ciudad de México. En seguida se presenta gráficamente la tendencia de la recolección de pilas.

\section{Gráfico 1. Comparativo anual de recolección de pilas}

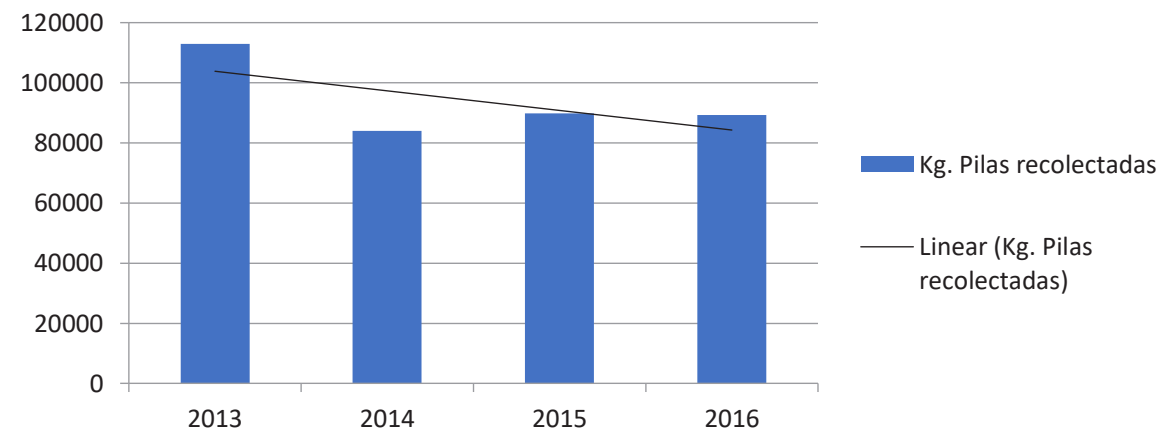

Fuente: Informes de resultados 2012-2016 Ponte las pilas

Además de mostrar la tendencia del programa, de acuerdo a la Gráfica 1, cabe señalar que las cantidades de pilas recolectadas representa en promedio $94050 \mathrm{kgs}$. al año y se mantiene un volumen constante entre los años 2013 al 2016, sin embargo, en 2016 hubo un decremento del 0.51\% respecto al 2013, situación que invita a reflexionar sobre las estrategias que se deben diseñar para incentivar y fomentar al consumidor sobre la disposición de este tipo de materiales y que éstos no contaminen.

Respecto al programa "Reciclatrón", los resultados observados desde su lanzamiento, son los siguientes: 


\section{Gráfico 2. Comparativo anual de recolección de residuos electrónicos}

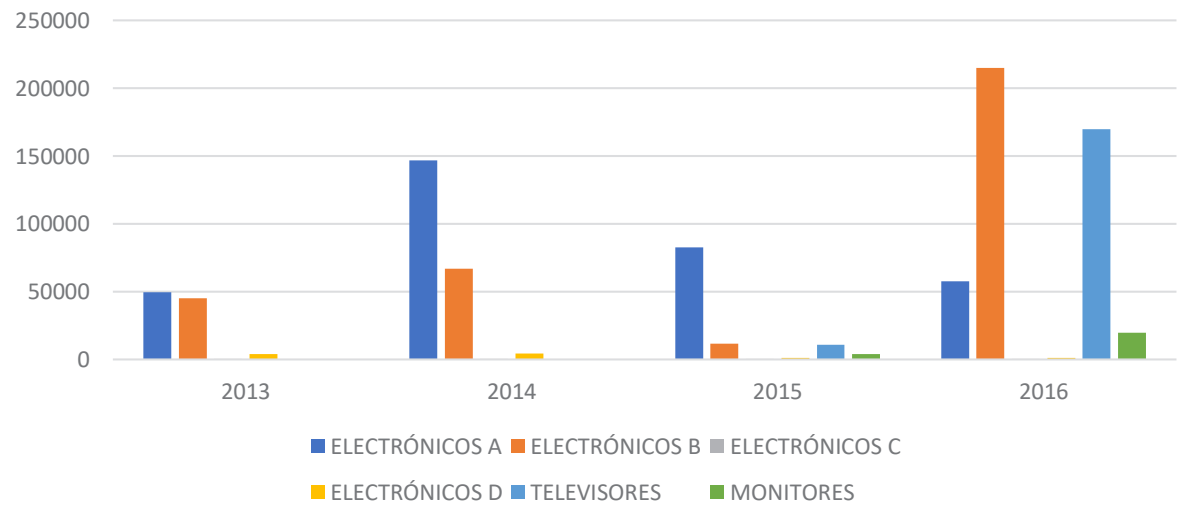

Fuente: Informes de resultados 2012-2016 Reciclatrón

De acuerdo a la Gráfica 2, los residuos sólidos recuperados en 2016 representaron el 9.8\% del total generado de RSU, cabe destacar que la recolecta con mayor consistencia de 2013 a 2016 es la clasificación de electrónicos tipo "A" que incluye teclados, impresoras, faxes, MP3, cámaras, escáner, radiograbadoras, teléfonos, electrodomésticos, asimismo el pico de recolección de este programa fue originado por el "apagón analógico”, en 2016 el Reciclatrón creció el 7.47\%, destacando la recepción de televisores y electrónicos " $\mathrm{B}$ ".

De acuerdo con los resultados obtenidos a lo largo de los últimos 5 años, el programa "mercado de trueque" se ha comportado de la siguiente manera: 


\section{Gráfico 3. Comparativo anual de recolección de residuos sólidos}

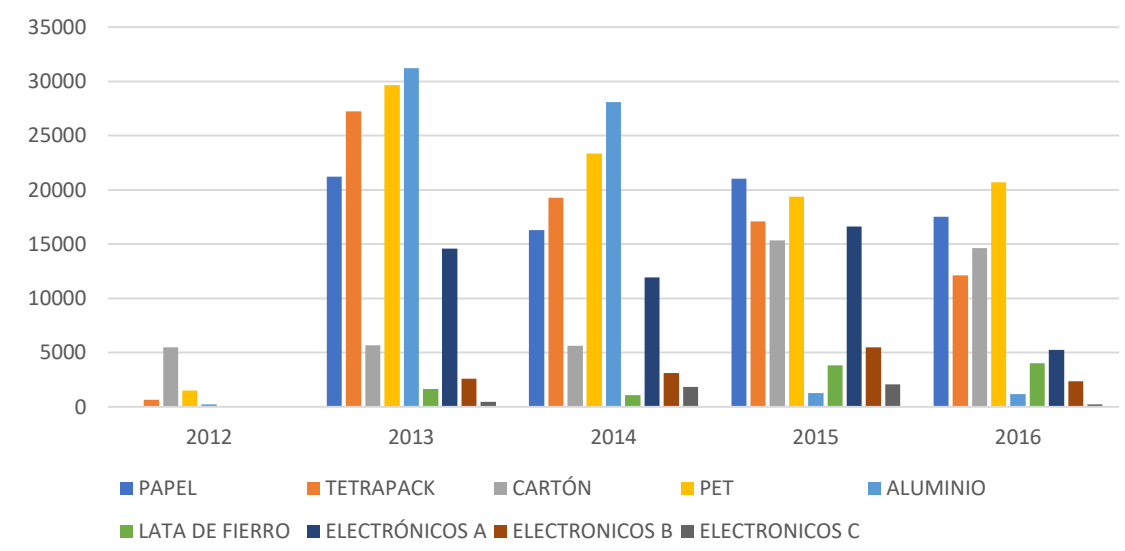

Fuente: Informes de resultados 2012-2016 Mercado del trueque

A partir de la Gráfica 3, es posible observar que la cantidad de residuos sólidos recuperados en este programa representan el $2.3 \%$ de la generación anual total de residuos sólidos. Particularmente en este programa, más que el incremento o reducción en la recolección de RSU, destaca la participación ciudadana, la cual, a pesar de incrementar de 3 mil asistentes en 2012 a más de 36, 000 asistentes en promedio. Respecto a la Gráfica 4, se puede observar que a partir de 2014 ha aumentado de manera importante la asistencia a los mercados de trueque.

\section{Gráfico 4. Comparativo de asistentes al Mercado de Trueque}

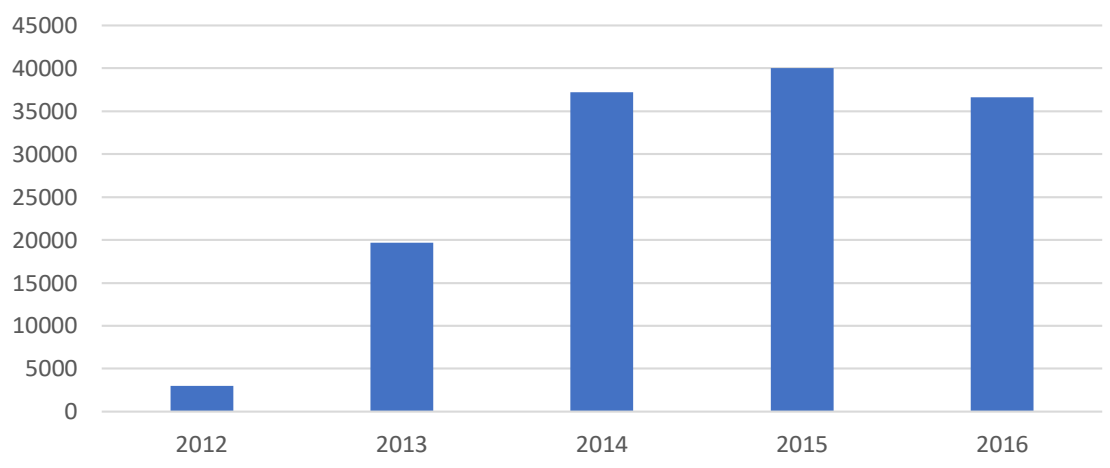

Fuente: Informes de resultados 2012-2016 Mercado del trueque 
Ahora bien, al agrupar los resultados obtenidos a lo largo de los últimos cinco años y enfocarlos únicamente a las categorías de los materiales de los que están hechos los empaques que protegen los productos agroindustriales, se puede observar el comportamiento de recolección:

\section{Gráfico 5. Comparativo de recolección de residuos empleados en envases de productos agroalimentarios}

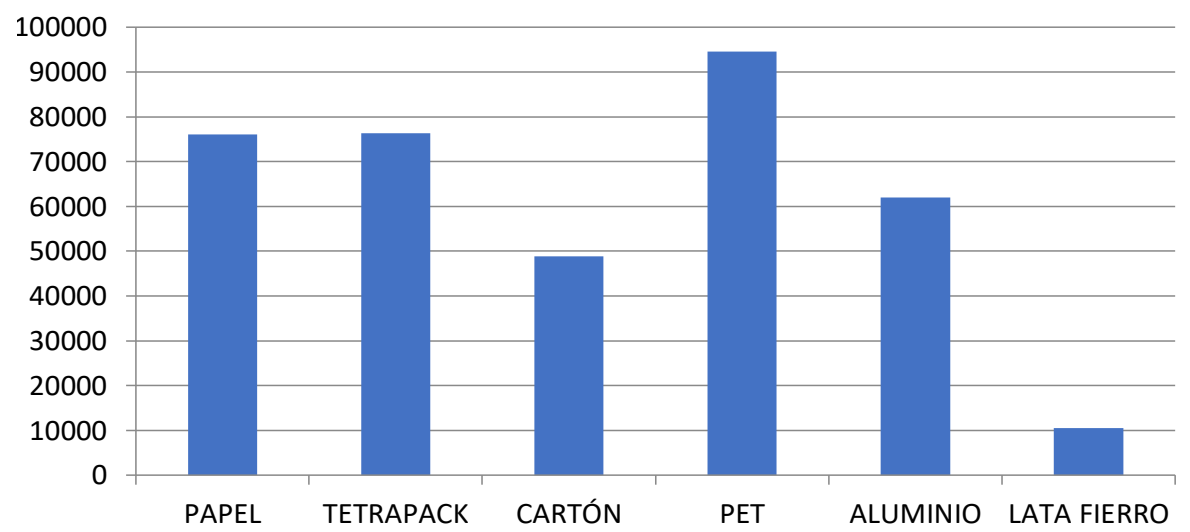

Fuente: Informes de resultados 2012-2016 Mercado del trueque

Donde, los materiales que más se recolectan son: el PET, tetrapack y papel; el correcto procesamiento de estos materiales tiene un gran impacto ecológico ya que tardan aproximadamente 30 y 100 años respectivamente en degradarse.

\section{Conclusiones}

A partir del análisis de los datos de los tres proyectos vigentes de la Ciudad de México, es viable observar que, para generar una cultura de reciclaje en los consumidores, influyen diversos factores como las políticas públicas, regulaciones, programas e incentivos que eduquen, guíen y fomenten el aprovechamiento de los residuos sólidos. La información que emanan los organismos públicos, privados y programas vigentes no es de fácil acceso para el público en general, además que la misma se presenta con diferentes indicadores y agrupamiento de residuos, lo que 
dificulta un análisis más profundo. En el sentido de la utilidad de la información ésta debe procurar persuadir a los consumidores con la finalidad de concientizarlos para que la participación sea constante y no temporal.

De los tres programas analizados, destaca el hecho de que en el año 2013, un año después de la publicación del PIGRS, existió mayor difusión de los programas y, de manera general, representó el año con mayor recaudación de RSU; esta situación muestra que los consumidores responden ante la influencia de factores externos, como la publicidad. De esta manera, es posible observar que programas como "Ponte las pilas" y "Mercado de trueque", que tienen una difusión constante, presentan resultados ascendentes.

Por otra parte, ha sido posible observar que los consumidores reaccionan positivamente ante los incentivos ya que en el Reciclatrón y Ponte las pilas, que no ofrecen ningún beneficio, los consumidores van perdiendo interés en participar conforme avanza el tiempo, en cambio, en el Mercado de trueque, que existe el intercambiar desechos sólidos por productos de consumo humano, los ha motivado a asistir al mercado y ampliar dicho programa

Finalmente, consideramos que mientras no exista un impulso a la difusión de los programas de reciclaje éstos no tendrán la eficacia que se requieren para incrementar la recolección de materiales y desechos que contaminan el ambiente (más rezagados). Si un programa de estos se posiciona en la mente del consumidor, entonces tendrá mayores resultados. Por ende, crear una cultura de reciclaje y consumo responsable no sólo requiere del consumidor o de la sociedad en general también dependerá de las acciones gubernamentales.

\section{Bibliografía}

Diario Oficial de la Federación, Ley General para la prevención y gestión de integral de los residuos, México, 2003, p. 52.

Franklin, Enrique. B., Krieger, Mario, Comportamiento Organizacional Enfoque para América Latina, Pearson, México, 2011, p. 550 . 
Gaceta Oficial de la Ciudad de México, Ley General para la Prevención y Gestión Integral de Residuos Sólidos, Ciudad de México, 2016, p. 62.

Gaceta Oficial de la Ciudad de México, Programa para la Gestión Integral de Residuos Sólidos, Ciudad de México, 2016 pp. 1-62.

Greenpeace, Información Básica sobre Pilas y Baterías, México, 2006, p. 6.

Hofstede, G, Culturas y Organizaciones: el software mental, Alianza, Madrid, 1999, pp. 3-31.

Martínez-Hernáez, A., El dibujante de límites: Franz Boas y la (im) posibilidad del concepto de cultura antropológica, Scielo, 2010 p. 16.

Mercado H., S., Comportamiento del consumidor, Grupo Vanchri, México, 2011, p. 676.

Profeco, Consumo y consumismo, Ciudad de México, 2013, p. 44.

Rodriguez Martinez, A., Antonio Aragón, J., Ávalos Ruiz, K.I., "Cultura de reutilización y reciclaje en estudiantes de humanidades de primer y tercer grados", Sociogénesis, 2010, pp. 4-6.

Roth Deubel, A.-N., Politicas Publicas Formulacion, Implementacion y Evaluacion, Aurora, Colombia, 2002, p.4.

SEDEMA, Inventario de Residuos Sólidos de la Ciudad de México, México, 2015, p. 114.

SEMARNAT, Fundación Flor y Canto. Educación y gestión escolar para el desarrollo sustentable: residuos sólidos y consumo responsable, Tierra Firme S.A. de C.V., México, 2012, p. 100.

UNESCO, Conferencia mundial sobre las politicas culturales, México, 1982, p. 6 . 


\section{Fuentes digitales}

SEDEMA, http://data.sedema.cdmx.gob.mx/sedema/index.php/ponte-las-pilas-con-tu-ciudad, [8 de mayo de 2017].

SEDEMA, http://data.sedema.cdmx.gob.mx/reciclatron/\#.WRBCBvk1_IU, [11 de mayo de 2017].

SEDEMA, http://data.sedema.cdmx.gob.mx/mercadodetrueque/, [19 de mayo 2017].

Fundación FIDA, Anta Martínez, María, http://www.ambientum.com/ revistanueva/2006-10/culturareciclaje.htm, [20 de abril de 2017]. 
